\title{
Clinical utility of recombinant adenoviral human p53 gene therapy: current perspectives
}

This article was published in the following Dove Press journal:

OncoTargets and Therapy

21 October 2014

Number of times this article has been viewed

\author{
Guang-xia Chen ${ }^{1, *}$ \\ Shu Zhang ${ }^{2-4, *}$ \\ Xiao-hua $\mathrm{He}^{\prime}$ \\ Shi-yu Liu' \\ Chao $\mathrm{Ma}^{2-4}$ \\ Xiao-Ping Zou ${ }^{2-4}$ \\ 'Department of Gastroenterology, \\ First People's Hospital of Xuzhou, \\ Xuzhou, Jiangsu Province, People's \\ Republic of China; ${ }^{2}$ Department \\ of Gastroenterology, Drum Tower \\ Hospital, ${ }^{3}$ Medical School of Nanjing \\ University, ${ }^{4}$ Jiangsu Clinical Medical \\ Center of Digestive Disease, Nanjing, \\ People's Republic of China \\ *These authors have contributed \\ equally to the paper
}

Correspondence: Xiao-Ping Zou Department of Gastroenterology, Drum Tower Hospital Affiliated to Medical School of Nanjing University, Nanjing 210008, People's Republic of China

Tel +86258830461620601

Fax $+862583 \mid 05206$

Email13770771661@I63.com

\begin{abstract}
Gene therapy has promised to be a highly effective antitumor treatment by introducing a tumor suppressor gene or the abrogation of an oncogene. Among the potential therapeutic transgenes, the tumor suppressor gene p 53 serves as an attractive target. Restoration of wild-type p53 function in tumors can be achieved by introduction of an intact complementary deoxyribonucleic acid copy of the p53 gene using a suitable viral vector, in most cases an adenoviral vector (Adp53). Preclinical in vitro and in vivo studies have shown that Adp53 triggers a dramatic tumor regression response in various cancers. These viruses are engineered to lack certain early proteins and are thus replication defective, including Gendicine, SCH-58500, and Advexin. Several types of tumor-specific p53-expressing conditionally replicating adenovirus vectors (known as replication-competent CRAdp53 vectors) have been developed, such as ONYX 015, AdDelta24-p53, SG600-p53, OBP-702, and H101. Various clinical trials have been conducted to investigate the safety and efficiency of these adenoviral vectors. In this review we will talk about the biological mechanisms, clinical utility, and therapeutic potentials of the replicationdeficient Adp53-based and replication-competent CRAdp53-based gene therapy.
\end{abstract}

Keywords: adenovirus, Adp53, CRAdp53

\section{Introduction}

Because cancer is an acquired genetic disorder, gene therapy promises to be a highly effective antitumor treatment for inducing cell death via introduction of a therapeutic tumor suppressor gene or the abrogation of an oncogene, which is tailored to the precise genetic structure of each tumor and therefore produces low systemic toxicity. ${ }^{1,2}$ Among the potential therapeutic transgenes, the tumor suppressor gene p53 is an attractive target because of its function as the "guardian of the genome" by inducing cell cycle arrest, senescence, and apoptosis in response to oncogene activation, deoxyribonucleic acid (DNA) damage, and other stress signals. ${ }^{3}$ Inactivation of p53 function is a common feature of human tumors that often correlates with increased malignancy, poor patient survival, and resistance to chemotherapy and/or radiotherapy. ${ }^{4-6}$ Evidence generated over the past decade has demonstrated that the p53 tumor suppressor is the most frequently altered gene in over $50 \%$ of all types of human cancers, with more than 25,000 mutations currently reported in the International Agency for Research on Cancer TP53 database. ${ }^{7}$ These findings suggest that $\mathrm{p} 53$ has potent and critical roles in suppressing malignant progression, making restoration of wild-type p53 gene function a promising antitumor strategy.

Restoration of wild-type p53 function in tumors can be achieved by introduction of an intact complementary DNA copy of the p53 gene using a suitable viral vector, in

submit your manuscript $\mid$ www. dovepress.con 
most cases an adenoviral vector (Adp53). ${ }^{8}$ This gene replacement therapy to transfer p53 gene directly into cancer cells has been demonstrated to suppress tumor growth because ectopic expression of exogenous p53 gene efficiently induces cell death and cell-cycle arrest in a variety of p53-inactivated tumor cells, with evidence for bystander effects in some cases. ${ }^{9}$ An advantage of the adenovirus delivery system is that it does not result in integration of the vector DNA into the host cells. ${ }^{10}$ Preclinical in vitro and in vivo studies have shown that Adp53 triggers a dramatic tumor regression response in various cancers, including head and neck cancer, ${ }^{11}$ lung cancer, ${ }^{12}$ colorectal cancer, ${ }^{13}$ ovarian cancer,${ }^{14}$ bladder cancer, ${ }^{15}$ prostate cancer, ${ }^{16}$ and esophageal cancer. ${ }^{17}$ These viruses are engineered to lack certain early proteins and are thus replication defective. With this method thousands of patients have received Adp53-based gene therapies in clinical trials, mostly in the USA and the People's Republic of China. Gendicine is the world's first Adp53-based gene therapy product approved by a government agency for clinical use. This recombinant human Adp53 injection developed by Shenzhen SiBiono Gene Tech (SiBiono; Shenzhen, People's Republic of China) was approved by the State Food and Drug Administration of the People's Republic of China (SFDA) on October 16, 2003 for the treatment of head and neck squamous cell carcinoma, and was formally launched in April 2004. ${ }^{18}$ Other Adp53 vectors such as SCH-58500 (CANJ, Inc., San Diego, CA, USA) and Advexin (INGN-201; Introgen Therapeutics, Inc. Austin, TX, USA) have been developed and used in various clinical trials. ${ }^{19-21}$ Regarding the low transduction rate of p53 gene introduction via Adp53 vector, several types of tumorspecific p53-expressing conditionally replicating adenovirus vectors (known as replication-competent CRAdp53 vectors) have been developed, such as ONYX 015, AdDelta24-p53, SG600-p53, OBP-702, and H10122-26 (Table 1). In this review, we will talk about the biological mechanisms, clinical utility, and therapeutic potentials of the replication-deficient Adp53-based and replication-competent CRAdp53-based gene therapy.

\section{Molecular mechanism}

As p53 activity is impaired or defective in most human cancers, the obvious goal of Adp53 vectors is to reestablish the growth-inhibitory functions of p53 in cancer cells. Cell death pathways, such as apoptosis and autophagy, induced by p53 expression are mainly involved in the suppression of tumor initiation and development. A great variety of genes are now identified as p53 target genes, whose protein products induce multiple outcomes such as apoptosis (eg, Bax, DR5, PUMA,
Table I Clinical studies of p53 cancer gene therapy using Adp53 and CRAdp53 vectors

\begin{tabular}{lll}
\hline $\begin{array}{l}\text { Type of adenoviral } \\
\text { vector }\end{array}$ & $\begin{array}{l}\text { Recombinant } \\
\text { adenovirus }\end{array}$ & Cancer types \\
\hline $\begin{array}{l}\text { Replication-deficient } \\
\text { Adp53 vectors }\end{array}$ & $\begin{array}{l}\text { Gendicine } \\
\text { Advexin }\end{array}$ & SCCHN, NSCLC, HCC \\
& SCH-58500 & NSCLC \\
Replication-competent & ONYX015 & SCCHN, colorectal cancer, \\
CRAdp3 vectors & & $\begin{array}{l}\text { pancreatic carcinoma, } \\
\text { malignant glioma, sarcomas }\end{array}$ \\
& HI0I & SCCHN \\
& AdDelta24-p53 & $/$ \\
& SG600-p53 & $/$ \\
& OBP-702 & $/$ \\
\hline
\end{tabular}

Note: ' $/$ ' indicates no data.

Abbreviations: HCC, hepatocellular carcinoma; NSCLC, non-small-cell lung cancer; SCCHN, squamous cell carcinoma of the head and neck.

and Noxa), cell cycle arrest (eg, p21 and GADD45), and autophagy (eg, DRAM ${ }^{27}$ (Figure 1). Several studies indicate that delivery of Adp53 induces dramatic cell death via apoptosis and cell cycle arrest in human glioma and glioblastoma cells, preceded by elevation in the levels of the p21 and Bax. ${ }^{28-30}$ OBP-702-mediated p53 transactivation demonstrates its therapeutic potential by inducing dual apoptotic and autophagic cell death pathways via regulation of DRAM, an

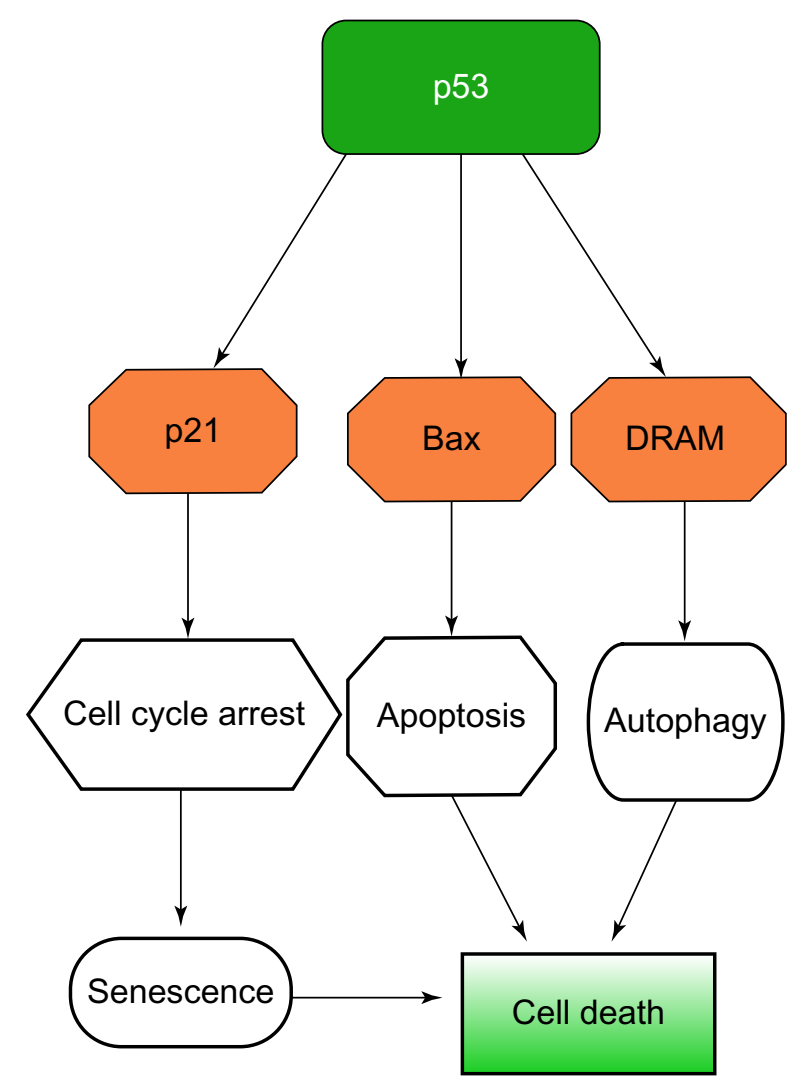

Figure I The schematic molecular mechanism of antitumor effect induced by Adp53-based gene therapy. 
important p53-induced modulator of autophagy, in human osteosarcoma cells. ${ }^{31}$ Because MDM2 functions as a negative regulator of $\mathrm{p} 53$ via ubiquitin-mediated p53 degradation, and induction of MDM2 expression can lead to resistance to p53 gene therapy for cancer, MDM2 suppression is thought to stabilize exogenous p53 expression. Cotransduction of ribosomal protein L23 can enhance the therapeutic efficacy of Adp53 gene transfer in models of human gastric cancer through inhibiting MDM2-mediated p53 degradation. ${ }^{32}$ What is more, suppression of $\mathrm{p} 21$ via $\mathrm{p} 21$-targeting micro-ribonucleic acids (microRNAs) can efficiently induce Adp53-mediated apoptosis and autophagy in human cancer cells. ${ }^{33}$

However, the inability of this approach to infect every cell in the tumor with virus has drawn people's attention. Problems of effective repeated dosing because of the presence of a host antibody to adenovirus that reduces their infectivity, also exist. Bystander effect is therefore very important in explaining Adp53-mediated inhibition of tumor progression. For example, Adp53 infection markedly inhibits the expression of an angiogenic factor, vascular endothelial growth factor, and increases the expression of an antiangiogenic factor, brain-specific angiogenesis inhibitor 1, resulting in reduced neovascularization and tumor growth of adjacent nontransduced cells in vivo. ${ }^{34}$ Immunological mechanisms involving natural killer cells play an important role in the bystander effect involving Adp53 gene therapy for ovarian cancer. Additionally, overexpression of CD95L by the wild-type p53 gene transfer induces neutrophil infiltration into human colorectal tumors. ${ }^{35}$ These findings suggest that Adp53-mediated p53 overexpression is a promising antitumor therapy because angiogenesis is inhibited and immune responses are involved within tumor tissues.

\section{Clinical utility of the replication- deficient Adp53-based gene therapy Gendicine}

Many clinical trials using Adp53-based therapies have been performed all over the world involving thousands of patients. As the first approved commercial Adp53 vector product, Gendicine, a recombinant human serotype 5 adenovirus in which the E1 region is replaced by a human wild-type p53 expression cassette, has been tested in Phase I to IV clinical trials for the treatment of patients with various cancers. The expressed $\mathrm{p} 53$ gene appears to exert its antitumor activities by triggering apoptotic pathways, activating immune response factors such as natural killer cells, inhibiting DNA repair and antiapoptosis functions, and blocking the transcription of survival signals.
In a Phase I clinical trial in 2003, Gendicine was used for treating 12 patients with advanced laryngeal cancer, with an average clinical course of 41 months. ${ }^{36}$ There was no patient relapse for more than 5 years after Gendicine treatment. In comparison, the 3 -year relapse rate for patients with advanced laryngeal cancer receiving surgery alone is generally about 30\%. Three Phase II/III clinical trials enrolling patients with squamous cell carcinomas of head and neck (SCCHN) demonstrated that Gendicine in combination with radiotherapy showed obvious synergistic effects. ${ }^{37-39}$ An extended follow-up on a subset of nasopharyngeal carcinoma patients, in which 82 patients were randomized to receive Adp53 injection and radiotherapy (GTRT; $n=42$ ) or radiotherapy alone (RT; $\mathrm{n}=40$ ), showed that complete response rate in the GTRT group was observed at 2.73 times that of the RT group (66.7\% vs $24.4 \%) .{ }^{40}$ Upregulation of $\mathrm{p} 21 / \mathrm{WAF} 1$ and Bax and downregulation of vascular endothelial growth factor were observed in postinjection tumor biopsy. Adp53 significantly increased the 5-year locoregional tumor control rate by $25.3 \%$ for patients treated with irradiation alone $(P=0.002)$. However, combination therapy did not significantly prolong survival and disease-free survival.

In a Phase I htrial of adenovirus p53 gene replacement for non-small-cell lung cancer (NSCLC), Adp53 was administered to 21 patients with advanced NSCLC and produced little toxicity, along with potentially useful clinical responses. ${ }^{41}$ Time to disease progression in the indicator lesion treated with Adp53 appears to be enhanced by higher doses of vector and concomitant cisplatin therapy. A nonrandomized, two-armed clinical trial enrolled 58 patients with advanced NSCLC, of which 19 received a combination therapy of bronchial arterial infusion and Gendicine (the combo group), while the remaining 39 were treated with only bronchial arterial infusion (the control group). ${ }^{42}$ The overall response rates were $47.3 \%$ and $38.4 \%$ for the combo and control group, respectively $(P>0.05)$. Patients in the combo group had a longer time to progression than those in the control group (a median 7.75 vs 5.5 months, $P=0.018$ ). But the combination treatment did not lead to better survival. In another Phase II trial, 40 patients diagnosed with NSCLC who had been treated with chemotherapy were recruited for this study. Nineteen of them were assigned to the DOCp53 group, receiving transtracheal Adp53 injection on Day 1 and Day 8, as well as docetaxel on Day 2, and 21 patients were assigned to the DOC group, receiving docetaxel alone on Day 1. Disappointingly, for patients with relapsed NSCLC, transtracheal injection of Adp53 in addition to docetaxel did not improve overall survival or efficacy. ${ }^{43}$ 
In 2005, a case report showed a 23-year-old patient with recurrent hepatocellular carcinoma (HCC) who was treated with Gendicine combined with transcatheter arterial chemoembolization (TACE). ${ }^{44}$ A total of $3 \times 10^{12}$ virus particles of Gendicine were infused via the hepatic artery. Four days later, the virus particles superselectively embolized the patient's hepatic arteries with 5-fluorouracil, vinorelbine, and iodized oil. Seven months later, the patient had normal liver function and was in good clinical health with no further recurrence. A clinical study in the People's Republic of China enrolled 150 patients, of whom 68 patients were treated with the Gendicine-TACE combination, and 82 patients were treated with TACE alone as a control group. ${ }^{18}$ The response rates were $67.6 \%$ in the Gendicine (GT)-TACE group and $51.2 \%$ in the TACE-alone group $(P<0.05)$. The 6-month survival rate was $76.5 \%$ in the GT-TACE group and only $23.2 \%$ in the control group $(P<0.01)$, showing that Gendicine in combination with TACE is effective for the treatment of HCC to enhance patients' survival rate. In another study, 40 patients with diagnosed primary $\mathrm{HCC}$ were randomly treated by fractionated stereotactic radiotherapy (fSRT group) alone or Gendicine combined with fSRT (combined group). ${ }^{45}$ These patients belonged to a group lower than Grade B on Child-Pugh classification with no extrahepatic metastasis. During 44 months of follow-up, the 1-year survival rates of the fSRT and combined groups were $70.0 \%$ and $90.0 \%$ respectively $(P=0.081)$, suggesting the potential efficacy of Gendicine combined with fSRT in treating patients with $\mathrm{HCC}$.

\section{Advexin}

Another Adp53 vector, Advexin, has not yet been approved by the Food and Drug Administration and the company developing it has closed, although some remarkable clinical cases have been reported. ${ }^{10}$ A Phase I/II study of Advexin enrolled ten patients with chemoradiation-resistant advanced esophageal squamous cell carcinoma. Intratumoral injections of Advexin were administered on Days 1 and 3 for up to five cycles. Local tumor responses revealed stable disease in nine cases and progressive disease in one case ${ }^{46}$ In another Phase II study, 13 patients with squamous cell carcinoma of the oral cavity, oropharynx, larynx, or hypopharynx received surgery and perioperative Advexin injections in the primary tumor bed and the ipsilateral neck. ${ }^{47}$ In addition, three patients received injections in the contralateral neck. This therapy presented to be technically feasible, and the estimate of 1-year progression-free survival was 92\%. Additional Phase III trials are warranted to enhance our understanding on the efficacy of Advexin in increasing the survival rates of patients with various malignancies.

\section{$\mathrm{SCH}-58500$}

Twenty-five patients with nonresectable NSCLC were enrolled in an open-label, multicenter Phase II study of three cycles of regimen A, carboplatin plus paclitaxel, or regimen B, cisplatin plus vinorelbine in combination with intratumoral injection of $7.5 \times 10^{12}$ particles of SCH-58500. ${ }^{48}$ There was no significant difference between the response rate of lesions treated with p53 gene therapy combined with chemotherapy (52\%) and lesions treated with chemotherapy alone (48\%). There was no survival difference between the two groups, and the median survival of the cohort was 10.5 months (1-year survival, 44\%). Conclusively the authors suggested that intratumoral SCH-58500 therapy appeared to provide no additional benefit in patients receiving chemotherapy for advanced NSCLC. Several other Phase I/II clinical trials have demonstrated that SCH-58500 therapy is a feasible and safe treatment for ovarian and bladder cancer. ${ }^{49,50}$ However, we are not able to conclude whether SCH-58500 has potential benefit on survival and tumor response rates of patients with various cancers.

\section{Safety and immune responses of the replication-deficient Adp53 in clinical use}

Although some self-limited fever cases have been reported, so far no severe adverse effects have been found in patients treated with Gendicine and other replication-deficient Adp53 vectors. No related fatalities have been reported. Data from the clinical trials have demonstrated that replication-deficient Adp53-based gene therapy is well tolerated and safe for clinical use.

The adenoviral vector is known to induce a strong immune response in patients, which is manifested by the development of self-limited fevers. Although considered to be a side effect in clinical use, fever also reflects the effectiveness and benefits of Adp53 in mobilizing the body's immune systems.

\section{Therapeutic potential and clinical utility of the replication-competent CRAdp53-based gene therapy}

The low transduction rate of p53 gene via Adp53 is a major problem that must be solved to improve the clinical outcomes of patients with various advanced cancers. The adenovirus E1B 55K protein binds and blocks p53, thus preventing 
p53-induced cell death and apoptosis, and allowing viral replication and production of viral progeny. ${ }^{8}$ Therefore, these tumor-specific, replication-competent CRAdp53 vectors have been developed in which the promoters of cancer-related genes are used to enhance E1-dependent virus replication in a tumor-dependent manner. ${ }^{51}$ As a consequence, CRAdp53 is superior to Adp53 vectors in inducing higher p53 expression and stronger antitumor effects through induction of cell death and apoptosis. Here we discuss the therapeutic potential of several types of CRAdp53 vectors (eg, ONYX 015, AdDelta24-p53, SG600-p53, OBP-301, OBP-702, and H101) in anticancer p53 gene therapies.

\section{ONYX 015}

Exploiting a virus with a known deletion in the E1B region of its genome, the Onyx company developed and brought into clinical trial the oncolytic ONYX 015 virus. ${ }^{10}$ ONYX 015 is currently the most prominent and clinically evaluated CRAdp 53 vector which can only proliferate effectively in $\mathrm{p} 53$ mutant but not p53 wild-type cells. However, this concept remains controversial. Some studies have demonstrated that wild-type $\mathrm{p} 53$ is necessary for the activation of the rapid adenovirus-induced cell death, ${ }^{52}$ or that ONYX 015 replication in human tumor cells does not correlate with the status of $\mathrm{p} 53 .{ }^{53}$ The current view indicates that ONYX 015 oncolytic selectivity seems to be defined not purely by $\mathrm{p} 53$ status but rather by the state of the stress response in the target cell. ${ }^{54,55}$

In an in vivo study, ONYX 015 was proved to inhibit tumor growth, and its antitumor efficacy combined with chemotherapy was significantly greater than either agent alone. ${ }^{56}$ RNA interference-mediated knockdown of p21 enhances antitumor efficacy of ONYX 015 in human cancer cells. ${ }^{57}$

Numerous clinical trials have been conducted among patients with various advanced cancers. In a Phase II trial, 37 patients with recurrent head and neck carcinoma were enrolled. ${ }^{58}$ Significant tumor regression $(>50 \%)$ was observed in $21 \%$ of evaluable patients, with no toxicity to injected normal peritumoral tissues. p53 mutant tumors were significantly more likely to undergo ONYX 015 -induced necrosis than p53 wild-type tumors. Another Phase II trial enrolled 40 patients with SCCHN, receiving ONYX 015 at a dose of 20 particles via intratumoral injection for either 5 consecutive days (standard, 30) or twice daily for two consecutive weeks (hyperfractionated, ten) during a 21-day cycle. ${ }^{59}$ Standard treatment resulted in $14 \%$ partial to complete regression, while hyperfractionated treatment resulted in $10 \%$ complete response, indicating that ONYX 015 was a safe treatment for SCCHN with evidence of modest antitumoral activity. ONYX 015 combined with chemotherapy is more efficient than chemotherapy alone for patients with recurrent SCCHN. ${ }^{60}$ ONYX 015 may also benefit patients with refractory metastatic colorectal cancer who have failed prior treatment with 5-fluorouracil (FU)/leucovorin by hepatic artery infusion. ${ }^{61}$ For patients with unresectable pancreatic carcinoma, ONYX 015 injection into tumors via endoscopic ultrasound by the transgastric route with prophylactic antibiotics was feasible and generally well tolerated either alone or in combination with gemcitabine. ${ }^{21}$ Two of the 21 patients had partial regression of the injected tumor, two had minor responses, and six had stable disease. Other Phase I/II trials showed that patients with advanced sarcomas, hepatobiliary tumors, and malignant gliomas could tolerate ONYX 015, alone or with other chemotherapy, well and safely. ${ }^{62-64}$ They also provided some evidence of antitumor activity for ONYX 015. However, the clinical effect of ONYX 015 has varied greatly, and there are still many patients responding poorly. The reason may have to do with the fact that E1B 55K has other functions than binding $\mathrm{p} 53$, including viral RNA export and inhibition of host protein synthesis, as previously described. The actual effects of ONYX 015 in treating various tumors need to be further investigated in preclinical experiments and clinical trials.

\section{$\mathrm{HIOI}$}

H101 is a recombinant human type 5 adenovirus with a total deletion of E1B 55K gene and an additional deletion of 78.3-85.8 $\mu \mathrm{m}$ gene segment in the E3 region, making it a CRAdp53 vector similar to ONYX $015 .{ }^{25}$ Deleted segments of E3 region may enhance the safety of the product. The oncolytic anticancer effect has been confirmed by various clinical trials, although the exact mechanism is not clear.

A Phase II clinical study conducted in the People's Republic of China enrolled 50 patients with malignant tumors in multiple centers, receiving H101 therapy $0.5 \mathrm{~mL} 50$ viral particles per day for 5 consecutive days every 3 weeks, combined with routine chemotherapy. ${ }^{65}$ The overall response rate was $30.4 \%$ among 46 valuable cases, with well-tolerated toxicities. A Phase III randomized clinical trial compared effects and toxicities of intratumoral H101 injection combined with cisplatin plus 5-fluorouracil (PF) regimen versus $\mathrm{PF}$ regimen alone in treating patients with head and neck or esophagus squamous cell cancer. ${ }^{66}$ Among 123 evaluable patients, the overall response rate of PF plus $\mathrm{H} 101$ group was $78.8 \%$, and of $\mathrm{PF}$ alone group was $39.6 \%$. Differences of response rates between the two groups were significant $(P=0.000)$. As a result, intratumoral injection of H101 showed efficacy in treating patients with squamous cell 
cancer of the head and neck or esophagus. Chinese government regulators (SFDA) approved H101 especially for advanced nasopharyngeal carcinoma in combination with cisplatin and 5 -FU chemotherapy in 2005. Recent in vitro studies demonstrate that the combination of H101 and dacarbazine exerts a synergistic antitumor effect to uveal melanoma cells without enhanced toxicity to normal cells via a type of cell cycle block, reflecting $\mathrm{H} 101$ a promising agent in treating melanoma. ${ }^{67}$

\section{Other CRAdp53 vectors}

AdDelta24-p53 is a CRAdp53 vector in which the Rb proteinbinding CR2 domain (24 bp) of the E1A region is deleted and the p53 expression cassette under the regulation of simian virus 40 early promoter is inserted into the E3 region. ${ }^{22}$ This virus expresses enhanced oncolytic potency on $80 \%$ of tested human cancer cell lines. AdDelta24-p53 can also kill most malignant glioma cell lines, inhibit glioma growth in animals, and prolong animals surviving in an in vivo study. ${ }^{68}$ This vector in combination with radiotherapy causes an increase in the percentage of apoptotic glioma cells and a significant enhancement of the number of mice demonstrating tumor regression as well as long-term survival. ${ }^{69}$ However, a small number of cancer cell lines expressing high levels of the major negative p53 regulator MDM2 respond poorly to AdDelta24-p53. So a novel CRAdp53 vector AdDelta24p53(14/19) encoding a p53 variant incapable of binding to MDM2 has been developed, exhibiting approximately ten times more effectively than AdDelta24-p53 in killing cancer cell lines with high levels of MDM2 expression. ${ }^{70}$ This finding suggests that inhibition of MDM2-dependent p53 negative regulation serves to be a promising treatment for increasing the antitumor efficacy of CRAdp53 gene therapy.

Another CRAdp53 vector SG600-p53 has been engineered, in which the E1A gene with a deletion of 24 nucleotides within $\mathrm{CR} 2$ region is controlled under the human telomerase reverse transcriptase promoter, and the E1B gene is directed by the hypoxia response element, whereas the p53 gene is controlled by the cytomegalovirus promoter. ${ }^{23}$ The tumor-selective replication of this vector and its antitumor efficacy are characterized in several tumor cell lines in vitro and in xenograft models of human non-small-cell lung cancer (NSCLC) in nude mice. Vincristine might increase the antitumor efficacy of SG600 against retinoblastoma cells. ${ }^{71}$ Recently, SG635-p53 has been developed in which the Ad5 fiber is replaced with a chimeric Ad5/35 fiber. This novel vector produces more progeny viruses, mediates a higher level of transgenic expression, and shows a markedly enhanced antitumor effect in HCC cells compared with SG600-p53. ${ }^{72}$
OBP-301 is an adenovirus 5 vector in which the human telomerase reverse transcriptase promoter element drives expression of E1A and E1B genes linked with an internal ribosome entry site. ${ }^{73}$ OBP-301 induces tumor-selective oncolysis and antitumor effect in a telomerase-dependent manner in various cancer cells; eg, head and neck cancer, human bone and soft tissue sarcomas, and prostate cancer. ${ }^{74-76}$ This vector injected into the primary tumor might be effective in purging micrometastasis from regional lymph nodes in an orthotopic colorectal cancer model. ${ }^{77}$ OBP-301 enhances the sensitivity of various cancers to chemotherapy; eg, lung cancer, ovarian cancer. ${ }^{78,79}$ Then OBP-702 is engineered by combining OBP-301 and wild-type p53 gene. OBP-702 suppresses the viability of both OBP-301-sensitive and OBP-301-resistant human cancer cells, inhibits tumor growth in mice models, and induces more profound cell apoptosis and autophagy compared to monotherapy with OBP-301 or Adp53. ${ }^{24,31}$ This effect is probably achieved through E1Adependent p21 and MDM2 suppression.

Although basic research has demonstrated the antitumor potential of these CRAdp53 vectors, we lack evidence from clinical trials to determine the safety, feasibility, and clinical efficacy of these vectors.

\section{Conclusion}

As a common tumor suppressing gene, p53 serves as a very important gene therapy target. Thus, adenovirus-mediated p53 cancer gene therapy develops and proves to be a promising antitumor strategy to restore the wild-type p53 function, inducing profound p53-mediated cell death pathways. In this review, we discuss the molecular mechanisms and preclinical and clinical studies of Adp53 and CRAdp53 gene therapy in many types of human cancers. Phase I/II/III clinical trials of some replication-deficient Adp53 vectors have been carried out in patients with diverse cancers, demonstrating that Adp53 gene therapy is well tolerated, feasible, and exerts promising antitumor effects. Regarding the low transduction rate and insufficient delivering system of these Adp53 vectors in many patients, more replication-competent CRAdp53 vectors have been engineered. However, clinical studies fail to provide enough evidence of safety and antitumor efficacy for these CRAdp53 vectors. Until now, only Gendicine and H101 have been approved by the government (SFDA), and these vectors, both Adp53 and CRAdp53, are not widely used for patients around the world. The exact mechanisms and clinical antitumor effect of these vectors need to be further explored. More safe and efficient delivering systems should be developed. 


\section{Acknowledgments}

This work was funded by the National Natural Science Foundation of China (Grant No 81101814, 81272742).

\section{Disclosure}

The authors declare no conflicts of interest in this work.

\section{References}

1. Roth JA, Cristiano RJ. Gene therapy for cancer: what have we done and where are we going? J Natl Cancer Inst. 1997;89(1):21-39.

2. Brenner MK, Gottschalk S, Leen AM, Vera JF. Is cancer gene therapy an empty suit? Lancet Oncol. 2013;14(11):e447-e456.

3. Brown CJ, Lain S, Verma CS, Fersht AR, Lane DP. Awakening guardian angels: drugging the p53 pathway. Nat Rev Cancer. 2009;9(12): 862-873.

4. Bassett EA, Wang W, Rastinejad F, El-Deiry WS. Structural and functional basis for therapeutic modulation of p53 signaling. Clin Cancer Res. 2008;14(20):6376-6386.

5. Munro AJ, Lain S, Lane DP. P53 abnormalities and outcomes in colorectal cancer: a systematic review. Br J Cancer. 2005;92(3):434-444.

6. Martins CP, Brown-Swigart L, Evan GI. Modeling the therapeutic efficacy of p53 restoration in tumors. Cell. 2006;127(7):1323-1334.

7. Lehmann BD, Pietenpol JA. Targeting mutant p53 in human tumors. J Clin Oncol. 2012;30(29):3648-3650.

8. Wiman KG. Restoration of wild-type p53 function in human tumors: strategies for efficient cancer therapy. Adv Cancer Res. 2007;97: 321-338.

9. Roth JA, Grammer SF. Tumor suppressor gene therapy. Methods Mol Biol. 2003;223:577-598.

10. Lane DP, Cheok CF, Lain S. p53-based cancer therapy. Cold Spring Harb Perspect Biol. 2010;2(9):a001222.

11. Clayman GL, el-Naggar AK, Roth JA, et al. In vivo molecular therapy with p53 adenovirus for microscopic residual head and neck squamous carcinoma. Cancer Res. 1995;55(1):1-6.

12. Fujiwara T, Grimm EA, Mukhopadhyay T, Zhang WW, OwenSchaub LB, Roth JA. Induction of chemosensitivity in human lung cancer cells in vivo by adenovirus-mediated transfer of the wild-type p53 gene. Cancer Res. 1994;54(9):2287-2291.

13. Spitz FR, Nguyen D, Skibber JM, Cusack J, Roth JA, Cristiano RJ. In vivo adenovirus-mediated p53 tumor suppressor gene therapy for colorectal cancer. Anticancer Res. 1996;16(6B):3415-3422.

14. Kim J, Hwang ES, Kim JS, You EH, Lee SH, Lee JH. Intraperitoneal gene therapy with adenoviral-mediated p53 tumor suppressor gene for ovarian cancer model in nude mouse. Cancer Gene Ther. 1999;6(2): 172-178.

15. Pagliaro LC, Keyhani A, Liu B, Perrotte P, Wilson D, Dinney CP. Adenoviral p53 gene transfer in human bladder cancer cell lines: cytotoxicity and synergy with cisplatin. Urol Oncol. 2003;21(6):456-462.

16. Yang C, Cirielli C, Capogrossi MC, Passaniti A. Adenovirus-mediated wild-type $\mathrm{p} 53$ expression induces apoptosis and suppresses tumorigenesis of prostatic tumor cells. Cancer Res. 1995;55(19):4210-4213.

17. Shimada H, Shimizu T, Ochiai T, et al. Preclinical study of adenoviral p53 gene therapy for esophageal cancer. Surg Today. 2001;31(7):597-604.

18. Peng Z. Current status of gendicine in China: recombinant human Ad-p53 agent for treatment of cancers. Hum Gene Ther. 2005;16(9):1016-1027.

19. Gabrilovich DI. INGN 201 (Advexin): adenoviral p53 gene therapy for cancer. Expert Opin Biol Ther. 2006;6(8):823-832.

20. Wills KN, Maneval DC, Menzel P, et al. Development and characterization of recombinant adenoviruses encoding human p53 for gene therapy of cancer. Hum Gene Ther. 1994;5(9):1079-1088.

21. Hecht JR, Bedford R, Abbruzzese JL, et al. A phase I/II trial of intratumoral endoscopic ultrasound injection of ONYX-015 with intravenous gemcitabine in unresectable pancreatic carcinoma. Clin Cancer Res. 2003;9(2):555-561.
22. van Beusechem VW, van den Doel PB, Grill J, Pinedo HM, Gerritsen WR. Conditionally replicative adenovirus expressing p53 exhibits enhanced oncolytic potency. Cancer Res. 2002;62(21): 6165-6171.

23. Wang X, Su C, Cao H, et al. A novel triple-regulated oncolytic adenovirus carrying p53 gene exerts potent antitumor efficacy on common human solid cancers. Mol Cancer Ther. 2008;7(6):1598-1603.

24. Yamasaki Y, Tazawa H, Hashimoto Y, et al. A novel apoptotic mechanism of genetically engineered adenovirus-mediated tumourspecific p53 overexpression through E1A-dependent p21 and MDM2 suppression. Eur J Cancer. 2012;48(14):2282-2291.

25. Yu W, Fang H. Clinical trials with oncolytic adenovirus in China. Curr Cancer Drug Targets. 2007;7(2):141-148.

26. Fujiwara T, Urata Y, Tanaka N. Telomerase-specific oncolytic virotherapy for human cancer with the hTERT promoter. Curr Cancer Drug Targets. 2007;7(2):191-201.

27. Stegh AH. Targeting the p53 signaling pathway in cancer therapy the promises, challenges and perils. Expert Opin Ther Targets. 2012; 16(1):67-83.

28. Gomez-Manzano C, Fueyo J, Kyritsis AP, et al. Adenovirus-mediated transfer of the p53 gene produces rapid and generalized death of human glioma cells via apoptosis. Cancer Res. 1996;56(4):694-699.

29. Gomez-Manzano C, Fueyo J, Kyritsis AP, et al. Characterization of p53 and p21 functional interactions in glioma cells en route to apoptosis J Natl Cancer Inst. 1997;89(14):1036-1044.

30. Köck H, Harris MP, Anderson SC, et al. Adenovirus-mediated p53 gene transfer suppresses growth of human glioblastoma cells in vitro and in vivo. Int J Cancer. 1996;67(6):808-815.

31. Hasei J, Sasaki T, Tazawa H, et al. Dual programmed cell death pathways induced by p53 transactivation overcome resistance to oncolytic adenovirus in human osteosarcoma cells. Mol Cancer Ther. 2013;12(3): 314-325.

32. Zhang YF, Zhang BC, Zhang AR, et al. Co-transduction of ribosomal protein L23 enhances the therapeutic efficacy of adenoviral-mediated p53 gene transfer in human gastric cancer. Oncol Rep. 2013;30(4): 1989-1995.

33. Idogawa M, Sasaki Y, Suzuki H, et al. A single recombinant adenovirus expressing p53 and p21-targeting artificial microRNAs efficiently induces apoptosis in human cancer cells. Clin Cancer Res. 2009;15(11): 3725-3732.

34. Nishizaki M, Fujiwara T, Tanida T, et al. Recombinant adenovirus expressing wild-type p53 is antiangiogenic: a proposed mechanism for bystander effect. Clin Cancer Res. 1999;5(5):1015-1023.

35. Waku T, Fujiwara T, Shao J, et al. Contribution of CD95 ligand-induced neutrophil infiltration to the bystander effect in p53 gene therapy for human cancer. J Immunol. 2000;165(10):5884-5890.

36. Han DM, Huang ZG, Zhang W, et al. [Effectiveness of recombinant adenovirus p53 injection on laryngeal cancer: phase I clinical trial and follow up]. Zhonghua Yi Xue Za Zhi. 2003;83(23):2029-2032. Chinese.

37. Zhang SW, Xiao SW, Liu CQ, et al. [Treatment of head and neck squamous cell carcinoma by recombinant adenovirus-p53 combined with radiotherapy: a phase II clinical trial of 42 cases]. Zhonghua $Y_{i}$ Xue Za Zhi. 2003;83(23):2023-2028. Chinese.

38. Chen CB, Pan JJ, Xu LY. [Recombinant adenovirus p53 agent injection combined with radiotherapy in treatment of nasopharyngeal carcinoma: a phase II clinical trial]. Zhonghua Yi Xue Za Zhi. 2003;83(23): 2033-2035. Chinese.

39. Zhang SW, Xiao SW, Liu CQ, et al. [Recombinant adenovirus-p53 gene therapy combined with radiotherapy for head and neck squamouscell carcinoma]. Zhonghua Zhong Liu Za Zhi. 2005;27(7):426-428. Chinese.

40. Pan JJ, Zhang SW, Chen CB, et al. Effect of recombinant adenovirusp53 combined with radiotherapy on long-term prognosis of advanced nasopharyngeal carcinoma. J Clin Oncol. 2009;27(5):799-804.

41. Roth JA, Swisher SG, Merritt JA, et al. Gene therapy for non-small cell lung cancer: a preliminary report of a phase I trial of adenoviral p53 gene replacement. Semin Oncol. 1998;25(3 Supp1 8):33-37. 
42. Guan YS, Liu Y, Zou Q, et al. Adenovirus-mediated wild-type $\mathrm{p} 53$ gene transfer in combination with bronchial arterial infusion for treatment of advanced non-small-cell lung cancer, one year follow-up. $J$ Zhejiang Univ Sci B. 2009;10(5):331-340.

43. Ning $X$, Sun $Z$, Wang $Y$, et al. Docetaxel plus trans-tracheal injection of adenoviral-mediated p53 versus docetaxel alone in patients with previously treated non-small-cell lung cancer. Cancer Gene Ther. 2011;18(6):444-449.

44. Guan YS, Liu Y, Zhou XP, Li X, He Q, Sun L. p53 gene (Gendicine) and embolisation overcame recurrent hepatocellular carcinoma. Gut. 2005;54(9):1318-1319.

45. Yang ZX, Wang D, Wang G, et al. Clinical study of recombinant adenovirusp53 combined with fractionated stereotactic radiotherapy for hepatocellular carcinoma. J Cancer Res Clin Oncol. 2010;136(4):625-630.

46. Shimada H, Matsubara H, Shiratori T, et al. Phase I/II adenoviral p53 gene therapy for chemoradiation resistant advanced esophageal squamous cell carcinoma. Cancer Sci. 2006;97(6):554-561.

47. Yoo GH, Moon J, Leblanc M, et al. A phase 2 trial of surgery with perioperative INGN 201 (Ad5CMV-p53) gene therapy followed by chemoradiotherapy for advanced, resectable squamous cell carcinoma of the oral cavity, oropharynx, hypopharynx, and larynx: report of the Southwest Oncology Group. Arch Otolaryngol Head Neck Surg. 2009;135(9):869-874.

48. Schuler M, Herrmann R, De Greve JL, et al. Adenovirus-mediated wild-type p53 gene transfer in patients receiving chemotherapy for advanced non-small-cell lung cancer: results of a multicenter phase II study. J Clin Oncol. 2001;19(6):1750-1758.

49. Buller RE, Runnebaum IB, Karlan BY, et al. A phase I/II trial of $\mathrm{rAd} /$ p53 (SCH 58500) gene replacement in recurrent ovarian cancer. Cancer Gene Ther. 2002;9(7):553-566.

50. Kuball J, Wen SF, Leissner J, et al. Successful adenovirus-mediated wild-type $\mathrm{p} 53$ gene transfer in patients with bladder cancer by intravesical vector instillation. $J$ Clin Oncol. 2002;20(4):957-965.

51. Tazawa H, Kagawa S, Fujiwara T. Advances in adenovirus-mediated p53 cancer gene therapy. Expert Opin Biol Ther. 2013;13(11): $1569-1583$

52. Dix BR, O'Carroll SJ, Myers CJ, Edwards SJ, Braithwaite AW. Efficient induction of cell death by adenoviruses requires binding of E1B55k and p53. Cancer Res. 2000;60(10):2666-2672.

53. Goodrum FD, Ornelles DA. p53 status does not determine outcome of E1B 55-kilodalton mutant adenovirus lytic infection. $J$ Virol. 1998;72(12):9479-9490.

54. O'Shea CC, Johnson L, Bagus B, et al. Late viral RNA export, rather than $\mathrm{p} 53$ inactivation, determines ONYX-015 tumor selectivity. Cancer Cell. 2004;6(6):611-623.

55. O'Shea CC, Soria C, Bagus B, McCormick F. Heat shock phenocopies E1B-55K late functions and selectively sensitizes refractory tumor cells to ONYX-015 oncolytic viral therapy. Cancer Cell. 2005;8(1): 61-74.

56. Heise C, Sampson-Johannes A, Williams A, McCormick F, Von Hoff DD, Kirn DH. ONYX-015, an E1B gene-attenuated adenovirus, causes tumor-specific cytolysis and antitumoral efficacy that can be augmented by standard chemotherapeutic agents. Nat Med. 1997;3(6):639-645.

57. Shiina M, Lacher MD, Christian C, Korn WM. RNA interferencemediated knockdown of p21(WAF1) enhances anti-tumor cell activity of oncolytic adenoviruses. Cancer Gene Ther. 2009;16(11):810-819.

58. Nemunaitis J, Ganly I, Khuri F, et al. Selective replication and oncolysis in p53 mutant tumors with ONYX-015, an E1B-55kD gene-deleted adenovirus, in patients with advanced head and neck cancer: a phase II trial. Cancer Res. 2000;60(22):6359-6366.

59. Nemunaitis J, Khuri F, Ganly I, et al. Phase II trial of intratumoral administration of ONYX-015, a replication-selective adenovirus, in patients with refractory head and neck cancer. J Clin Oncol. 2001;19(2):289-298.

60. Khuri FR, Nemunaitis J, Ganly I, et al. a controlled trial of intratumoral ONYX-015, a selectively-replicating adenovirus, in combination with cisplatin and 5-fluorouracil in patients with recurrent head and neck cancer. Nat Med. 2000;6(8):879-885.
61. Reid TR, Freeman S, Post L, McCormick F, Sze DY. Effects of Onyx015 among metastatic colorectal cancer patients that have failed prior treatment with 5-FU/leucovorin. Cancer Gene Ther. 2005;12(8): 673-681.

62. Chiocca EA, Abbed KM, Tatter S, et al. A phase I open-label, dose-escalation, multi-institutional trial of injection with an E1BAttenuated adenovirus, ONYX-015, into the peritumoral region of recurrent malignant gliomas, in the adjuvant setting. Mol Ther. 2004;10(5):958-966.

63. Makower D, Rozenblit A, Kaufman H, et al. Phase II clinical trial of intralesional administration of the oncolytic adenovirus ONYX-015 in patients with hepatobiliary tumors with correlative p53 studies. Clin Cancer Res. 2003;9(2):693-702.

64. Galanis E, Okuno SH, Nascimento AG, et al. Phase I-II trial of ONYX015 in combination with MAP chemotherapy in patients with advanced sarcomas. Gene Ther. 2005;12(5):437-445.

65. Lu W, Zheng S, Li XF, Huang JJ, Zheng X, Li Z. Intra-tumor injection of $\mathrm{H} 101$, a recombinant adenovirus, in combination with chemotherapy in patients with advanced cancers: a pilot phase II clinical trial. World J Gastroenterol. 2004;10(24):3634-3638.

66. Xia ZJ, Chang JH, Zhang L, et al. [Phase III randomized clinical trial of intratumoral injection of E1B gene-deleted adenovirus (H101) combined with cisplatin-based chemotherapy in treating squamous cell cancer of head and neck or esophagus]. Ai Zheng. 2004;23(12): 1666-1670. Chinese.

67. Cun B, Song X, Jia R, et al. Combination of oncolytic adenovirus and dacarbazine attenuates antitumor ability against uveal melanoma cells via cell cycle block. Cancer Biol Ther. 2012;13(2):77-84.

68. Geoerger B, Vassal G, Opolon P, et al. Oncolytic activity of p53expressing conditionally replicative adenovirus AdDelta24-p53 against human malignant glioma. Cancer Res. 2004;64(16):5753-5759.

69. Idema S, Lamfers ML, van Beusechem VW, et al. AdDelta24 and the p53-expressing variant AdDelta24-p53 achieve potent anti-tumor activity in glioma when combined with radiotherapy. $J$ Gene Med. 2007;9(12):1046-1056.

70. van Beusechem VW, van den Doel PB, Gerritsen WR. Conditionally replicative adenovirus expressing degradation-resistant p53 for enhanced oncolysis of human cancer cells overexpressing murine double minute 2. Mol Cancer Ther. 2005;4(6):1013-1018.

71. Song X, Wang H, Jia R, et al. Combined Treatment with an Oncolytic Adenovirus and Antitumor Activity of Vincristine against Retinoblastoma Cells. Int J Mol Sci. 2012;13(9):10736-10749.

72. Chen W, Wu Y, Liu W, et al. Enhanced antitumor efficacy of a novel fiber chimeric oncolytic adenovirus expressing p53 on hepatocellular carcinoma. Cancer Lett. 2011;307(1):93-103.

73. Kawashima T, Kagawa S, Kobayashi N, et al. Telomerase-specific replication-selective virotherapy for human cancer. Clin Cancer Res. 2004;10(1 Pt 1):285-292.

74. Nakajima O, Matsunaga A, Ichimaru D, Urata Y, Fujiwara T, Kawakami K. Telomerase-specific virotherapy in an animal model of human head and neck cancer. Mol Cancer Ther. 2009;8(1):171-177.

75. Sasaki T, Tazawa H, Hasei J, et al. Preclinical evaluation of telomerase-specific oncolytic virotherapy for human bone and soft tissue sarcomas. Clin Cancer Res. 2011;17(7):1828-1838.

76. Huang P, Watanabe M, Kaku H, et al. Direct and distant antitumor effects of a telomerase-selective oncolytic adenoviral agent, OBP-301, in a mouse prostate cancer model. Cancer Gene Ther. 2008;15(5):315-322.

77. Kojima T, Watanabe Y, Hashimoto Y, et al. In vivo biological purging for lymph node metastasis of human colorectal cancer by telomerasespecific oncolytic virotherapy. Ann Surg. 2010;251(6):1079-1086.

78. Liu D, Kojima T, Ouchi M, et al. Preclinical evaluation of synergistic effect of telomerase-specific oncolytic virotherapy and gemcitabine for human lung cancer. Mol Cancer Ther. 2009;8(4):980-987.

79. Takakura M, Nakamura M, Kyo S, et al. Intraperitoneal administration of telomerase-specific oncolytic adenovirus sensitizes ovarian cancer cells to cisplatin and affects survival in a xenograft model with peritoneal dissemination.Cancer Gene Ther. 2010;17(1):11-19. 
OncoTargets and Therapy

\section{Publish your work in this journal}

OncoTargets and Therapy is an international, peer-reviewed, open access journal focusing on the pathological basis of all cancers, potential targets for therapy and treatment protocols employed to improve the management of cancer patients. The journal also focuses on the impact of management programs and new therapeutic agents and protocols on
Dovepress

patient perspectives such as quality of life, adherence and satisfaction. The manuscript management system is completely online and includes a very quick and fair peer-review system, which is all easy to use. Visit http://www.dovepress.com/testimonials.php to read real quotes from published authors.

\footnotetext{
Submit your manuscript here: http://www.dovepress.com/oncotargets-and-therapy-journal
} 